\title{
Motivation and Joy Mapping for Sustainable Healthy Lifestyle Design
}

\author{
Tabya Sultan' ${ }^{1}$ and Nitish $\mathrm{Nag}^{1}$
}

${ }^{1}$ Katal Think Labs, California, USA.

\begin{abstract}
Traditionally health systems focus on disease prevention through solutions designed around a population. A key challenge in this endeavor is the implementation of healthy daily actions towards one's desired health state. These everyday actions, commonly referred to as lifestyle, include repeated events such as eating, sleeping, moving, and mental preoccupations. Individuals may want to shape their lifestyle to be healthy and have the knowledge, logistical support, and resources to make this change; however, there is a gap between wanting and executing a sustainable method for implementing the desired lifestyle. Sustainability towards healthy living requires the best utilization of one's motivation and joy. We developed a matrix to organize intrinsic and extrinsic drivers that play a role in understanding the user's own behavioral patterns. In addition, leveraging the types of joy (hedonic versus eudaimonic) experienced by these daily actions can further enhance sustainability of healthy lifestyle actions. We distribute user actions into a matrix which allows for an organized viewpoint of a person's lifestyle. The lifestyle actions within this matrix are then attached with user specific weights for priority ranking. This ranked list is applied towards a more quantitative, personalized, and effective lifestyle design.
\end{abstract}

Keywords: health, well-being, wellness, lifestyle, lifestyle design, intrinsic and extrinsic motivation, joy.

\section{Introduction}

Historically, approaches to health and well-being within the United States healthcare system have been formulaic and one-size-fits-all solutions that focus on prevention from disease. Individuals are categorized as "normal," "at risk," or "diseased." Major modifiable risk factors, such as lifestyle, are major determinants of chronic diseases over time Organization 2020).

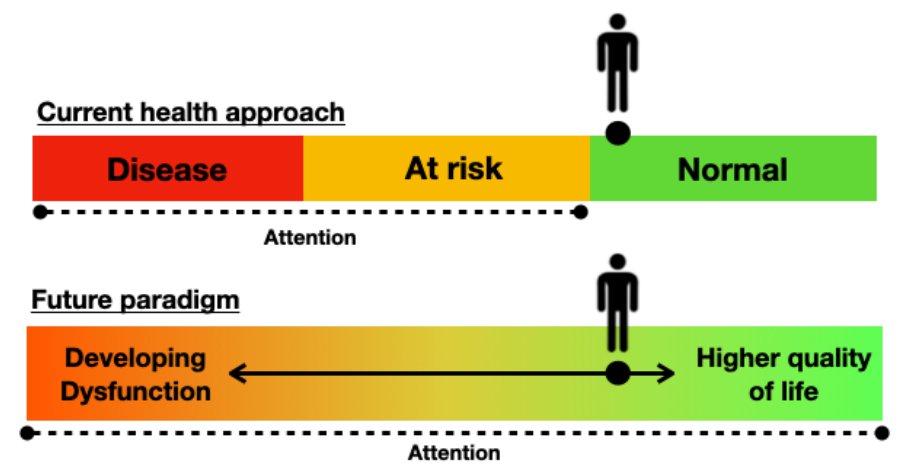

Figure 1. Health is a dynamic state that moves on a continuum. The attention placed on health should span the full spectrum, including high quality of living, or the "green," rather than just the diseased (red) or prodromal

Katal Think Labs (2020)

DOI: 10.31231/osf.io/5en2r

Corresponding author Tabya Sultan (yellow) states.

There are general recommendations or guidelines for the full population to make lifestyle changes to move from the red or dysfunctional health to the level of mediocre at-risk health (Figure 1). For example, the Centers for Disease Control and Prevention (CDC) highlights effective strategies 
for living a heart-healthy lifestyle to keep people from metabolic disease (CDC 2020). However, these population models based on disease prevention are not sufficiently effective for sustainable lifestyle change. Much of the change needed for improving lifestyle is dependent on personalized methods to move people towards a spectrum of higher quality living, not just keeping them in a mediocre state (Nag and Jain 2019). Effective lifestyle change depends on one's personalized life journey, including the quality of one's motivations (Linmans, Knottnerus, and Spigt 2015; Sebire et al. 2018). This conceptual shift towards increasing one's quality of life is highlighted by the health continuum in Figure 1 (Nag and Jain 2019).

Generally, individuals want to live long, free from disease, and with function to live independently (Venkatapuram 2013; Windsor 2009). To continuously increase the quality of life, there needs to be a clear connection between one's desires and actions. This connection can be made by first understanding the importance of health to an individual through their motivations. Once individuals establish motivations, they can then effectively design actions in their lifestyle that move their health state towards living a higher quality life.

We understand individuals want to be healthy through the growing demand in the health and wellness industry. A large developing area within modern approaches to holistic health is through various modes of wellness, including the consideration of inputs of lifestyle, the environment, and social and perceptual factors. Together, these factors bring about a more robust consideration of health in daily living and behavioral considerations, and an improvement in health performance, rather than an emphasis on eliminating or reducing disease. The economic size of this field globally is 4.5 trillion US Dollars, which is more than half as large as total global health expenditures at 7.3 trillion US dollars. It also represents 5.3 percent of global economic output (Global Wellness Institute 2016).

The wellness industry targets improvement in various components of an individual's life (CBInsights 2019). Many of the services provided involve tracking and developing methods for changing the health state. These include personalized nutrition, gym access, mental wellness via meditation and mindfulness apps, corporate wellness programs, sleep technologies, organic food, wellness travel, athleisure wear, and more. Various metrics demonstrate individual actions taken to be healthy; however, the approaches may not be sustainable. For example, according to statistics from U.S. News, 80 percent of New Year's resolutions are forgotten by February, leading to the cancellation of gym membership and lack of active engagement (Schmaltz 2018). People are motivated but have difficulty capitalizing on this motivation in the long term, demonstrating that resources are not the bottleneck for a significant part of the population.

These motivations and internal desires of an individual are most critical to address sustainable lifestyle change, given sufficient resources (Nag, Lux, and Jain 2018). This paper contributes a method by which to bridge the gap between wanting better health and implementing a healthier lifestyle through understanding, organizing, and using personal motivation and joy. Each individual will be considered a user of the system presented.

\section{Motivation and Joy as Fundamental Lifestyle Design Components}

Motivation reflects a user's enthusiasm and willingness to take action and guide behaviors. Identifying human motivations is needed for sustainable behavioral adaptation for developing an effective personalized lifestyle. We must look into the social-psychological factors in health. Motivations, such as specific health motivations, guide behavior and actions, as widely discussed by the Health Belief Model (Wiley and Cory 2013; Gochman 1972). Motivation theories such as the self-determination theory (SDT), suggest underlying psychological needs are essential for healthy functioning and well-being, outlining the framework of the study of intrinsic motivation (Ryan and Deci 2000; Deci and Ryan 2008). These needs are also described in Maslow's Hierarchy of Needs, proposing that humans must have social needs, esteem, and self-actualization for full functioning 


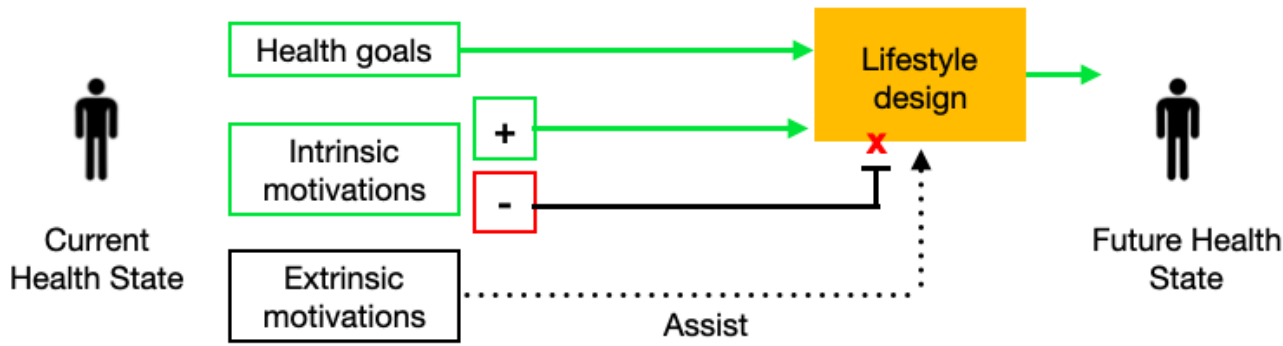

Figure 2. In order for the user to move from their current health state to the desired future health state, they need to design a lifestyle using their health goals and intrinsic motivations that are positive contributors to their health. The extrinsic motivations serve as assistance to help enhance the process.

(Badash et al. 2017). The psychological motivations rest on basic physiologic needs and safety as a baseline. We see further recognition of motivation in the Protection Motivation Theory, highlighting that users are more highly motivated if there is a threat to their health (Prentice-Dunn and Rogers 1986).

\subsection{Organizing Motivations: Intrinsic Versus Extrinsic Drivers}

A strategy to organize motivations has been to identify two types of motivations, extrinsic and intrinsic. Intrinsic motivations are the human propensity to learn, absorb, and inherently enjoy a process regardless of external rewards and pressures. Extrinsic motivations reflect external control and involve taking action due to a separable outcome (Deci and Ryan 2010). These may be external incentives such as materialistic rewards and punishment.

Users who are intrinsically motivated generally are engaged in something they enjoy and have a sense of mission. When in their healthiest states, users are active, given intrinsically motivated behaviors that result in acquiring knowledge about themselves (Deci and Ryan 2010). Intrinsically motivated users have a considerable advantage in that they are performing and acting on their health for their own sake and personal excellence. Developing intrinsic motivations can create focus, less fluctuation, and consistency in the approach, and decreased stress and distractions (Murphy 2005).

As health has been approached traditionally with a disease model, people interact with their health and healthcare professionals when in danger of entering the red zone, as shown in Figure 1. Instead of repairing the user, a sustainable healthy lifestyle design must look at users as continuously improving their health and quality of life (Seligman 2004). The interventions and approaches for lifestyle design must consider the user's joyful processes in the pursuit of their life's goals.

Extrinsic motivations may be driven by outcomes that are external to a user's internal gratification and enjoyment, leading to distractions or negative impact by external events. For example, if the user is motivated to "look fit," they may not enjoy the process of becoming fit, and thus the motivation may not be sustainable. Studies show that purely extrinsic motivations, such as weight loss for aesthetics, may not have positive psychological changes (Jackson et al. 2014). Similarly, external rewards or carrots such as receiving points or having recognition on badges for healthy actions may be heavily influenced socially or by monetary reward, and not sustainable for longterm behavior. Studies have also found that intrinsic motivations decrease when individuals are presented with external rewards (Deci 1971).

While extrinsic motivations may not be sufficient on its own, both intrinsic and extrinsic motivations can jointly play a role in changing the user's lifestyle, as shown in Figure 2 . For example, the user may enjoy the process of running and engaging in an outdoor environment. They may need an extrinsic goal, such as taking part in a half marathon, to push themselves and be held accountable. 


\subsection{Organizing Joy: Hedonia versus Eudaimonia}

Current research in positive psychology on well-being has two general perspectives on enjoyment (Ryan and Deci 2001). First is the hedonic adaptation, which focuses on happiness and defines well-being in terms of pleasure attainment and associated with emotions that gradually lose their intensity (Lyubomirsky 2007). An example of this is a user enjoying the food they are eating due to the taste. The second is the eudaimonic approach, which focuses on meaning and self-realization. It defines well-being in terms of the degree to which the user is fully functioning (Ryan and Deci 2000). The fulfilling nature of what clarity brings to the user is often eudaimonic, or "human flourishing and prosperity" based on long term fulfillment (Seligman 2004). Seligman and associates highlight that the greatest life satisfaction is the combination of the pursuit of meaning beyond oneself, finding a flow state, and partaking in life's pleasures. This principle can apply to develop the user's lifestyle in a way that brings the most sustainable, long-term joy in the process, or durable happiness (Lyubomirsky 2007). Durable happiness, or the sustainable happiness model, is determined by a genetically determined set point, life circumstances, and intentional activities that are driven by one's consciousness and effortful activities that increase happiness (Kurtz and Lyubomirsky 2008). The durability, or long-lasting nature of one's activities, is therefore a critical component alongside motivation and joy. Durability can be determined by evidence through the user's experience and how much they have consistently felt positively engaged in particular activities.

When driven by intrinsically motivated activities, users may find themselves in a flow state. The flow state is a genuinely satisfying experience, in which people are highly involved, engaged, and experience deep enjoyment (Csikszentmihalyi 1998). These states may be optimal experiences that can allow users to adequately and sustainably engage in an activity, such as mountain biking or trail running. Identifying and capitalizing on flow states is beneficial in designing lifestyle and choosing activities that allow for high level of engagement.

\section{Motivation and Joy Mapping Method}

We will discuss a motivation and joy mapping method that can be applied towards building healthy events. As discussed in this paper, motivation and joy mapping is a tool built for a healthy lifestyle, using a user's motivations, joyful processes, and the durability to drive lifestyle design. Our method allows users to understand their motivations and organize them into a framework to incorporate into their life design. It is important to filter these motivations to satisfy one's biological needs. For example, if the user is intrinsically motivated to smoke cigarettes, this may negatively impact their physical health state. The user must filter motivations for ensuring there is no harm to their health. This process is shown in Algorithm 1.

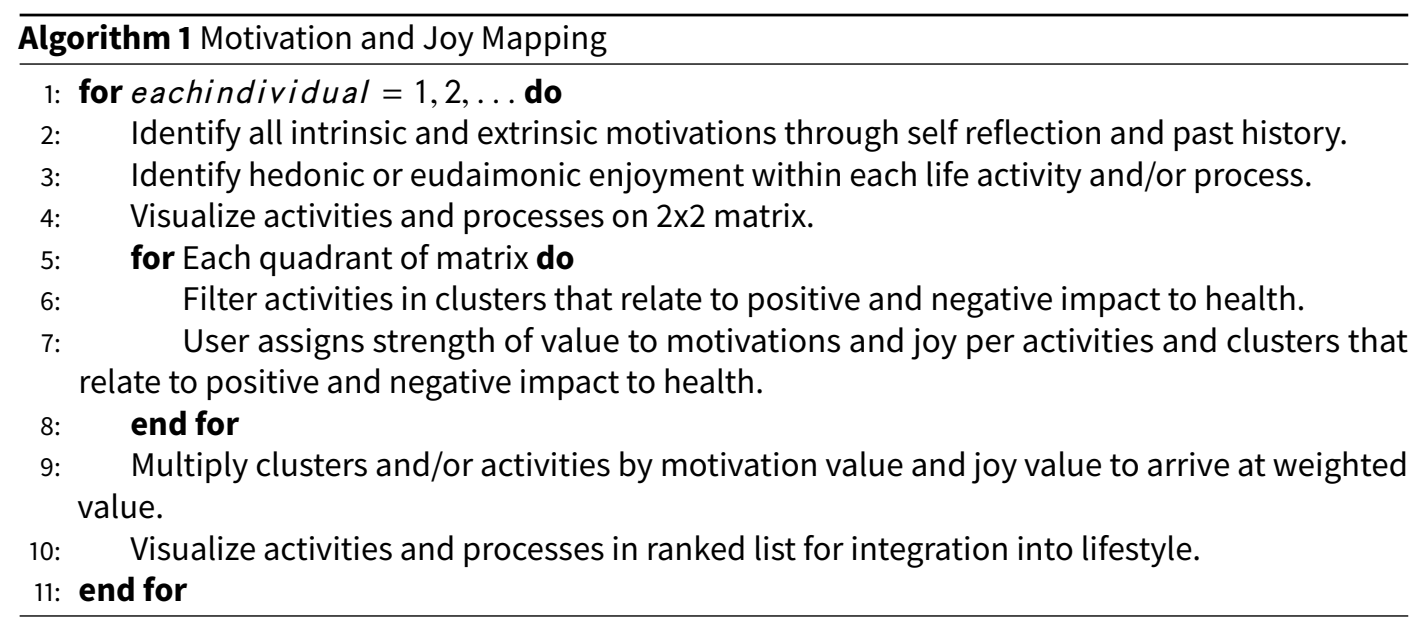


The components that we are considering in the mapping process are intrinsic and extrinsic motivations, eudaimonic and hedonic enjoyment, and positive and negative contributors to physical health. We can see this visually as an example in Figure 3. The map is divided by quadrants and within each quadrant are clusters reflecting positive and negative contributors to health.

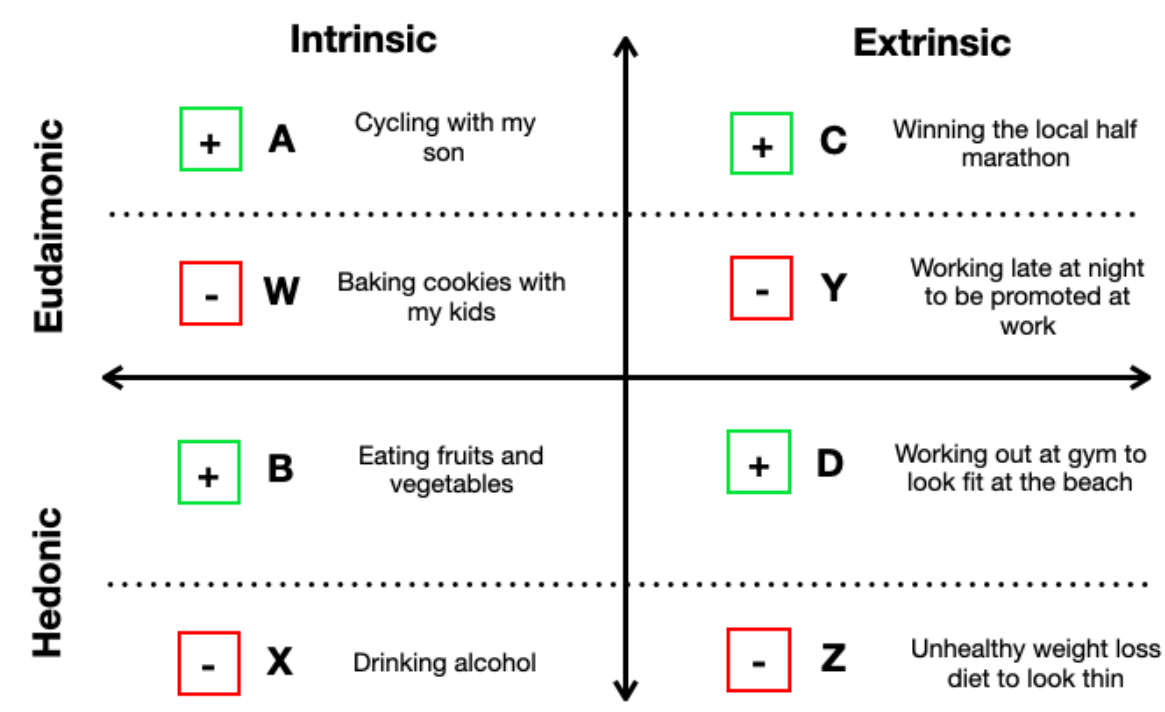

Figure 3. Motivation and joy mapping involves identifying the user's intrinsic and extrinsic motivations, eudaimonic and hedonic enjoyment, and positive and negative contributors to physical health. We see positive contributors identified as Clusters A, B, C, and D. Negative contributors are Clusters W, Y, X, and Z. Within the clusters are specific examples that are understandable to the user.

After the user has included all activities to relevant quadrants, they then add a subjective weight by perceived strength of value in motivation, joy, and durability, as shown in Table 1. The durability is determined using past data about the user to verify that the activity will be strong in the lifestyle design. For example, if the user has historically enjoyed trail running for the prior 10 years, we can conclude that trail running is a highly durable activity. The user assigns a subjective weight to each activity from a scale of 1 through 5 . In Table 1, we observe two different users who have unique personalities and perceptions of what activities are important. Users who place greater value on particular activities will determine the strength of the activity in the lifestyle design. We also include an objective multiplier depending on the quadrant the activity falls in. For example, intrinsically driven activities will have a $2 x$ multiplier while extrinsically driven activities will have a $1 x$ multiplier, due to the sustainability of the motivation type. We apply the same rationale to the multipliers for eudaimonic versus hedonic joy. 


\begin{tabular}{llllllll}
\hline & Cluster & Motivation (1-5) & M-Type & Joy (1-5) & J-Type & Durability (1-5) & Weight \\
\hline User 1 & A & 5 & 2 & 5 & 2 & 5 & $\mathbf{5 0 0}$ \\
& B & 5 & 2 & 3 & 1 & 4 & $\mathbf{1 2 0}$ \\
& C & 2 & 1 & 2 & 2 & 2 & $\mathbf{1 6}$ \\
& D & 2 & 1 & 1 & 1 & 1 & $\mathbf{2}$ \\
& & & & & & & \\
User 2 & A & 4 & 2 & 1 & 2 & 2 & $\mathbf{3 2}$ \\
& B & 4 & 2 & 3 & 1 & 3 & $\mathbf{7 2}$ \\
& C & 2 & 1 & 1 & 2 & 1 & $\mathbf{4}$ \\
& D & 3 & 1 & 2 & 1 & 1 & $\mathbf{6}$ \\
\hline
\end{tabular}

Table 1. The mapping tool requires individuals to assign a weight to each activity based on their perceived motivation, joy, and durability from a scale of 1 to 5 . User priorities may differ based on personality types and unique needs, as shown by the varying responses by Users 1 and 2. The M-Type is the motivation type, which has a $2 x$ multiplier for intrinsic motivations and $1 x$ multiplier for extrinsic motivations. The J-Type is the joy type, which has a $2 x$ multiplier for eudaimonic joy and $1 x$ multiplier for hedonic joy. The maximum weight on an activity is 500 . For User 1 , the activity priority ranking by weight to incorporate into the user's lifestyle design will be A, B, C, then D. For User 2, the priority ranking will be B, A, D, then C.

\section{Conclusion}

We must address health in the lens not just for disease prevention, but for higher quality of living, which begins with understanding what a high quality of life is for the user. Lifestyle change for sustainable healthy living is not a simple problem but is a critical need for moving people along the health continuum through everyday actions. The motivation and joy mapping method presented can be used to build more robust wellness systems that deliver personalized content and actionable insights, depending on the user's unique interests. The health and wellness industry must consider highly individualized approaches as the first step for desired mental and physical health outcomes. The method described in this paper is an initial framework that can be expanded upon with further complexities.

\section{Supplementary Material}

For supplementary material accompanying this paper, please visit www.katal.life

\section{References}

Badash, I., N. P. Kleinman, S. Barr, J. Jang, S. Rahman, and B. W. Wu. 2017. "Redefining Health: The Evolution of Health Ideas from Antiquity to the Era of Value-Based Care." Cureus (February). ISSN: 2168-8184. doi:10. 7759/cureus.1018.

CBInsights. 2019. Global Healthcare Report Q3 2019. https://www.cbinsights.com/research/report/healthcaretrends-q3-2019/.

CDC. 2020. Division of Nutrition, Physical Activity, Overweight and Obesity (DNPAO). https://www.cdc.gov/ nccdphp/dnpao/index.html.

Csikszentmihalyi, M. 1998. Optimal Experience: Psychological Studies of Flow in Consciousness. Cambridge University Press. 
Deci, E. L. 1971. "Effects of externally mediated rewards on intrinsic motivation.” Journal of Personality and Social Psychology 18, no. 1 (April): 105-115. ISSN: 00223514. doi:10.1037/h0030644.

Deci, E. L., and R. M. Ryan. 2008. "Self-determination theory: A macrotheory of human motivation, development, and health.” In Canadian Psychology, 49:182-185. 3. August. doi:10.1037/a0012801.

. 2010. “Intrinsic Motivation.” The Corsini Encyclopedia of Psychology (Hoboken, NJ, USA) (January): 1-2. doi:10.1002/9780470479216.corpsy0467. http://doi.wiley.com/10.1002/9780470479216.corpsy0467.

Global Wellness Institute. 2016. Statistics \& Facts - Global Wellness Institute. https://globalwellnessinstitute. org/press - room/statistics - and - facts/\%20http:// www. globalwellnessinstitute.org/press-room/ statistics-and-facts/.

Gochman, D. S. 1972. "The organizing role of motivation in health beliefs and intentions.” Journal of health and social behavior 13 (3): 285-293. ISSN: 00221465. doi:10.2307/2136765. https://www.jstor.org/stable/ 2136765.

Jackson, S. E., A. Steptoe, R. J. Beeken, M. Kivimaki, and J. Wardle. 2014. "Psychological changes following weight loss in overweight and obese adults: A prospective cohort study." PLOS ONE 9, no. 8 (August). ISSN: 19326203. doi:10.1371/journal.pone.0104552.

Kurtz, J. L., and S. Lyubomirsky. 2008. Toward a Durable Happiness. Technical report.

Linmans, J. J., J. A. Knottnerus, and M. Spigt. 2015. "How motivated are patients with type 2 diabetes to change their lifestyle? A survey among patients and healthcare professionals." Primary Care Diabetes 9, no. 6 (December): 439-445. ISSN: 18780210. doi:10.1016/j.pcd.2015.02.001.

Lyubomirsky, S. 2007. The How of Happiness: A New Approach to Getting the Life You Want. New York, NY: Penguin Books.

Murphy, S. 2005. "The Sport Psych Handbook.” Human Kinetics Inc.

Nag, N., and R. Jain. 2019. "A Navigational Approach to Health: Actionable Guidance for Improved Quality of Life.” Computer 52 (4): 12-20. ISSN: 0018-9162.

Nag, N., M. Lux, and R. Jain. 2018. "Intrinsic and Extrinsic Motivation Modeling Essential for Multi-Modal Health Recommender Systems.” doi:10.1145/nnnnnnn.nnnnnnn. https://doi.org/10.1145/nnnnnnn.nnnnnnn.

Organization, W. H. 2020. Facing the Facts \#1 chronic diseases and their common risk factors. Technical report. World Health Organization. www.who.int/chp.

Prentice-Dunn, S., and R. W. Rogers. 1986. "Protection Motivation Theory and preventive health: beyond the Health Belief Model." Health Education Research 1 (3). https://academic. oup.com/her/articleabstract/1/3/153/608633.

Ryan, R. M., and E. L. Deci. 2001. "On Happiness and Human Potentials: A Review of Research on Hedonic and Eudaimonic Well-Being." Annual Review of Psychology 52, no. 1 (February): 141-166. ISSN: 0066-4308. doi:10.1146/annurev.psych.52.1.141.

2000. "Intrinsic and Extrinsic Motivations: Classic Definitions and New Directions." Contemporary Educational Psychology 25:54-67. doi:10.1006/ceps.1999.1020.

Schmaltz, J. 2018. How to Keep Resolutionists from Busting Your Gym's Bottom Line | IHRSA. https://www.ihrsa. org/improve-your-club/how-to-keep-resolution-busters-from-busting-your-gyms-bottom-line/.

Sebire, S. J., Z. Toumpakari, K. M. Turner, A. R. Cooper, A. S. Page, A. Malpass, and R. C. Andrews. 2018. “I've made this my lifestyle now: A prospective qualitative study of motivation for lifestyle change among people with newly diagnosed type two diabetes mellitus.” BMC Public Health 18, no. 1 (January): 204. ISSN: 14712458. doi:10.1186/s12889-018-5114-5. https://bmcpublichealth.biomedcentral.com/articles/10.1186/s12889018-5114-5.

Sultan, Nag. | Katal Think Labs 
Seligman, M. 2004. Authentic Happiness: Using the New Positive Psychology to Realize Your Potential for Lasting Fulfillment. New York, NY: Simon / Schuster.

Venkatapuram, S. 2013. "Health, vital goals, and central human capabilities." Bioethics 27, no. 5 (June): 271-279. ISSN: 02699702. doi:10.1111/j.1467-8519.2011.01953.x.

Wiley, D., and A. Cory. 2013. "Health Belief Model." In Encyclopedia of School Health. SAGE Publications. doi:10. 4135/9781452276250.n106.

Windsor, T. D. 2009. "Persistence in goal striving and positive reappraisal as psychosocial resources for ageing well: A dyadic analysis." Aging and Mental Health 13, no. 6 (November): 874-884. ISSN: 13607863. doi:10. 1080/13607860902918199. 